\title{
X-ray detections of two young bona-fide brown dwarfs *
}

\author{
H. Bouy
}

\author{
Max Planck für Extraterrestrische Phyisk, Giessenbachstraße, 85748 Garching bei München, Germany \\ e-mail: hbouy@mpe.mpg.de \\ Laboratoire d'Astrophysique de l'Observatoire de Grenoble, 414 rue de la piscine, 38400 Saint Martin d'Hères, France \\ European Southern Observatory, Karl Schwarzschildtstraße 2, 85748 Garching bei München, Germany
}

Received 6 January 2004 / Accepted 17 April 2004

\begin{abstract}
I report the detection of two bona-fide brown dwarfs by XMM-Newton: [GY92] 141 in the $\rho$-Ophiuchus star-forming region and DENIS J155601-233809 in the Upper Scorpius OB association. The two objects have been detected with luminosities of $L_{\mathrm{X}}=8.35 \pm 2.86 \times 10^{28} \mathrm{erg} \mathrm{s}^{-1}$ and $L_{\mathrm{X}}=6.54 \pm 1.35 \times 10^{28} \mathrm{erg} \mathrm{s}^{-1}$ respectively, corresponding to luminosity ratios of $\log \left(L_{\mathrm{X}} / L_{\mathrm{bol}}\right)=-2.07$ and $\log \left(L_{\mathrm{X}} / L_{\mathrm{bol}}\right)=-2.69$. The two sources were close to the limit of detection of the instruments at only $2 \sim 3-\sigma$ above the background level, and no significant flare or variation could be detected during the $48.3 \mathrm{ks}$ and $33.9 \mathrm{ks}$ observations. [GY92] 141 had already been observed 10 months earlier with Chandra by Imanishi et al. (2001) with a luminosity $\sim 14$ times fainter than the one reported here, indicating that the X-ray emission of this object is strongly variable.
\end{abstract}

Key words. stars: low-mass, brown dwarfs - stars: coronae - X-rays: individuals: [GY92] 141, DENIS J155601-233809

\section{Introduction}

Brown dwarfs are very low mass objects unable to sustain stable nuclear reactions. Although these objects are extremely cool and their atmospheres fully convective, they appear to be able to display some X-ray coronal activity. Several surveys in the field or in star forming regions using ROSAT (Mokler \& Stelzer 2002; Neuhäuser et al. 1999; Neuhauser \& Comeron 1998; Fleming 1998), XMM-Newton (Imanishi et al. 2003; Stelzer \& Neuhäuser 2003; Martín \& Bouy 2002) or Chandra (Wolk 2003; Tsuboi et al. 2003; Preibisch \& Zinnecker 2002; Adams et al. 2002; Imanishi et al. 2001; Rutledge et al. 2000; Fleming et al. 2000) lead to the identification of several ultracool and brown dwarfs as faint X-ray emitters, with typical luminosities of $L_{\mathrm{X}} \sim 10^{27 \sim 28} \mathrm{erg} \mathrm{s}^{-1}$, and some of them displaying strong variability. These observations suggest that there is apparently no drop in the X-ray luminosity at the substellar boundary. Although these X-ray features are likely to have a magnetic origin, the standard $(\alpha-\omega)$ dynamo cannot be at work because of the absence of a radiative core and the fully convective nature of these objects. Küker \& Rüdiger (1999) suggested that the magnetic field could be generated by a socalled $\alpha^{2}$-dynamo effect, where the magnetic field is produced by the action of the Coriolis force on the convective zones of the atmosphere. The X-ray emission might then be correlated to the rotation, and therefore the age, of the object, which is

* Based on observations obtained with the ESA XMM-Newton Satellite, reported in the The First XMM-Newton Serendipitous Source Catalogue, XMM-Newton Survey Science Centre (SSC), 2003. still not clear as shown by the X-ray detection of old lateM dwarfs in the field (Martín \& Bouy 2002; Rutledge et al. 2000; Fleming et al. 2000; Fleming 1998). Finally, since no abrupt changes are observed at the convective transition, the $\alpha^{2}$-dynamo would probably be present in the convective zones of more massive stars. The study of the X-ray activity of ultracool and brown dwarfs will therefore bring extremely important results not only on their own characteristics but also on the properties of more massive objects.

In this paper, I present the detection of two young brown dwarfs with the ESA XMM Newton satellite. In the first section, the two objects and their optical and infrared properties are presented; in Sect. 3 the observations are described, then in Sect. 4 a description is given of the data processing and analysis and finally in Sect. 5 the results are analyzed.

\section{Two young brown dwarfs}

The two objects have been confirmed as brown dwarfs and as members of the Upper Scorpius and $\rho$-Oph star forming regions by spectroscopic measurements. In this section, I will summarize the properties of these objects as reported in the literature.

\subsection{DENIS J155601-233809}

DENIS J155601-233809 has been reported as an M 6.5 dwarf and as a member of the Upper Scorpius OB association by Martín et al. (2004) on the basis of spectroscopic measurements. Proper motion measurements confirm that the object belongs to the association (Bouy et al., in prep.). 
Table 1. Astrometric and photometric measurements available for the two objects.

\begin{tabular}{|c|c|c|c|c|}
\hline$\overline{\mathrm{RA}^{1}}$ & $\overline{\operatorname{Dec}^{1}}$ & Filter & Mag & Source \\
\hline \multicolumn{5}{|c|}{ DENIS J155601-233809 } \\
\hline \multirow[t]{3}{*}{155601.04} & -233808.1 & $J$ & $13.86 \pm 0.03$ & 2MASS \\
\hline & & $H$ & $13.24 \pm 0.02$ & 2MASS \\
\hline & & $K$ & $12.81 \pm 0.03$ & 2MASS \\
\hline \multirow[t]{3}{*}{155601.04} & -233808.1 & $I$ & $16.32 \pm 0.07$ & DENIS \\
\hline & & $J$ & $13.96 \pm 0.11$ & DENIS \\
\hline & & $K$ & $12.85 \pm 0.14$ & DENIS \\
\hline \multicolumn{5}{|c|}{ GY 141} \\
\hline \multirow[t]{3}{*}{162651.28} & -243242 & $\bar{J}$ & $15.30 \pm 0.04$ & 2MASS \\
\hline & & $H$ & $14.47 \pm 0.05$ & 2MASS \\
\hline & & $K$ & $13.89 \pm 0.06$ & 2MASS \\
\hline \multirow[t]{3}{*}{162651.31} & -243242.9 & $I$ & $18.42 \pm 0.18$ & DENIS \\
\hline & & $J$ & $15.33 \pm 0.15$ & DENIS \\
\hline & & $K$ & $13.47 \pm 0.18$ & DENIS \\
\hline
\end{tabular}

${ }^{1} \mathrm{~J} 2000$.

Martín et al. (2004) report a clear $\mathrm{H} \alpha$ emission with an equivalent width of $E W(\mathrm{H} \alpha)=-20 \pm 3 \AA$, not strong enough to indicate significant accretion, but indicating that DENIS-P J155601-233809 is chromosphericaly active (see e.g. Barrado y Navascues \& Martín 2003 or Jayawardhana et al. 2003 for a discussion on $\mathrm{H} \alpha$ emission as an indicator of accretion and activity).

DENIS J155601-233809 was also reported in the 2MASS catalogue as 2MASS J155601-233808. Table 1 gives an overview of the photometric and astrometric properties of DENIS J155601-233809. DENIS and 2MASS photometry (see Table 1) indicate an $I-J$ colour of $2.46 \pm 0.10 \mathrm{mag}$. If we assume an intrinsic colour for M 6.5 dwarfs of $I-J=2.5-2.7 \mathrm{mag}$ as reported by Dahn et al. (2002), the measured $I-J$ colour corresponds to almost zero extinction $A_{\mathrm{V}} \sim$ 0 mag. Using the bolometric correction $B_{K}=2.95 \pm 0.15 \mathrm{mag}$ evaluated for M 6.5 dwarfs by Leggett et al. (2002), the 2MASS apparent magnitude $m_{K}=12.81 \pm 0.03 \mathrm{mag}$, and assuming a distance of $145 \pm 2 \mathrm{pc}$ as measured with Hipparcos by de Zeeuw et al. (1999), I arrive at $\log L / L_{\odot}=-2.08 \pm 0.05$.

\section{2. [GY92] 141}

[GY92] 141 (hereafter GY 141) was reported by Luhman et al. (1997) as a M 8.0 dwarf and as member of the $\rho$-Oph star forming region ( $\rho-O$ ph 162349.8-242601) using spectroscopic measurements. On 1997 April 14-15th they report relatively strong $\mathrm{H} \alpha$ emission with an equivalent width of $E W(\mathrm{H} \alpha) \sim-60 \AA$. On 2002 October 31 Jayawardhana et al. (2002) report $\mathrm{H} \alpha$ emission with an equivalent width of $E W(\mathrm{H} \alpha)=-13.4 \pm 0.2 \AA$. The variability and the strength of this emission suggest chromospheric activity, but part of it could be due to accretion. The detection of mid-infrared excess emission by Comeron et al. (1998) with a magnitude of $12.25 \pm 0.3 \mathrm{mag}$ at $4.5 \mu \mathrm{m}$ in the LW1 filter of ISOCAM indeed indicates the presence of circumstellar material.
Luhman et al. (1997) estimated a mass of 0.01-0.06 $M_{\odot}$ using the evolutionary tracks of Burrows et al. (1997) and Baraffe et al. (1997), and Gorlova et al. (2003) estimated a mass of $0.024 \pm 0.015 M_{\odot}$. GY 141 is therefore clearly substellar.

GY 141 has already been observed twice in X-rays, first in September 1994 with ROSAT by Neuhäuser et al. (1999) who did not detect it with an upper limit on the luminosity of $\log L_{X} \leq 27.86$, and second by Imanishi et al. (2001) with Chandra in May 2000, who detected it with a luminosity $L_{\mathrm{X}}=0.25 \times 10^{28} \mathrm{erg} / \mathrm{s}$ and $\log \left(L_{\mathrm{X}} / L_{\mathrm{bol}}\right)=-3.6$. The source was too faint to allow them to do spectroscopy. They also estimated the chance of a background source falling within their $9^{\prime \prime}$ beam to be $0.6 \%-1 \%$.

GY 141 was also reported in the DENIS catalogue as DENIS J162651.3-243242.9 and in the 2MASS catalogue as 2MASS J16265113-243242. Table 1 gives an overview of the photometric and astrometric properties of this object as reported in these two catalogues.

\section{Observations}

The two sources were reported in the XMM-Newton Serendipitous Source Catalogue (XMM-Newton Survey Science Centre 2003). By doing a cross-matching between a list of currently know brown dwarfs and brown dwarf candidates with the catalogue, two X-ray sources were found to have been reported at the position of these two objects. The corresponding data have been retrieved from the XMM-Newton Public Archive (program 0112380101, P.I. Turner, and program 0111120201, P.I. Watson) for further analysis.

\subsection{DENIS J155601-233809}

DENIS J155601-233809 was observed on 2000 August 26th for $48309 \mathrm{~s}$. The pointing was made at RA $=15^{\circ} 56^{\prime} 25.00^{\prime \prime}$ and Dec $=-23 \mathrm{~h} 37^{\prime} 47.02^{\prime \prime}$, which is $331^{\prime \prime}$ away from DENIS J155601-233809. The observation was done in prime full window mode with the medium filter, which prevents optical contamination from point sources as bright as $m_{v}=8 \sim$ 10 mag. Both MOS and PN detectors have a $27^{\prime}$ diameter field of view. DENIS J155601-233809 appears only on the EPIC PN and MOS2 images. It is reported in the XMM-Newton Serendipitous Source Catalogue as 1XMM J155601.1-233809. Figure 1 shows the combination of the EPIC PN, MOS1 and MOS2 images and the position of DENIS J155601-233809. For more details on XMM-Newton and its instruments, please refer to the XMM-Newton Users' Handbook (Ehle et al. 2003).

The X-ray source lies 1'.0 away from the DENIS and the 2MASS coordinates, therefore well within the absolute astrometric uncertainties of the pointing ( 4 " on average, according to the XMM-Newton Users' Handbook). No other optical/near infrared counterpart can be found in the DENIS or 2MASS catalogues within an area of $\sim 20^{\prime \prime}$ around this position, which makes it very likely that the faint X-ray source is associated with the DENIS object. Moreover, and as will be discussed in Sect. 4, the associated flux is consistent with that of a brown dwarf. The corrected detection likelihood value reported in the 


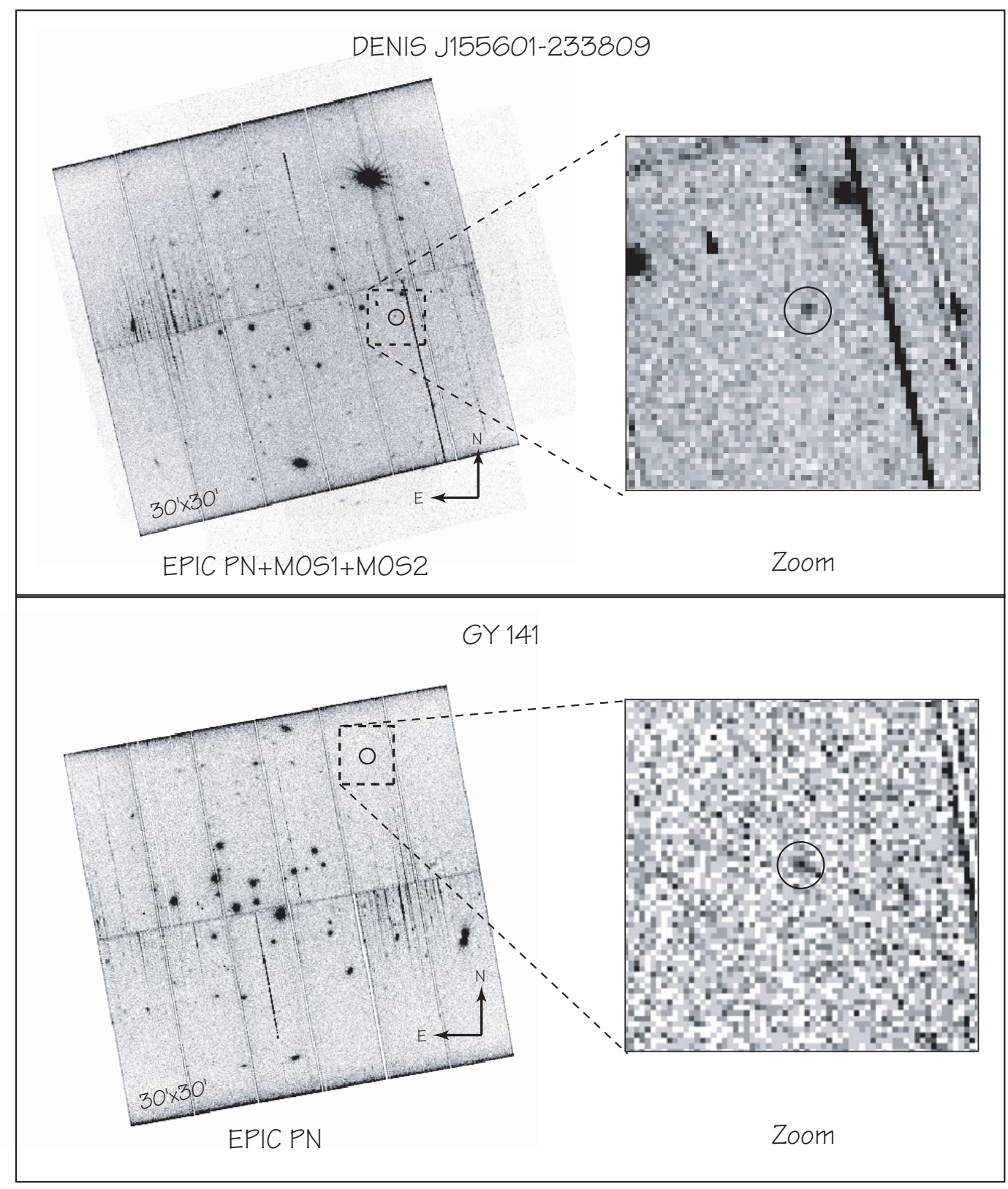

Fig. 1. This figure shows the XMM-Newton images where the two objects have been detected. For DENIS J155601-233809 it shows the combination of the images obtained with the 3 instruments (EPIC MOS1, MOS2 and PN cameras) and for GY 141 only the EPIC PN image.

catalogue is 44.8 , with a quality flag of 4 , ensuring that the probability that the detection is real is very high ${ }^{1}$.

\section{2. $G Y 141$}

GY 141 was observed on 2001 February 19th for 33908 s. The pointing was made on the core of the $\rho$-Oph star forming region, 722" away from GY 141. This observation was

\footnotetext{
1 According to the User Guide to the Catalogue, "the likelihood value stands for the detection likelihood of the source, $L=-\ln (p)$, where $p$ is the probability of the detection occurring by chance. A value of $\sim 7$ corresponds roughly to one false detection per exposure. $A$ summary quality flag of 4 indicates that the detection is good $(0=$ bad; $1-3=$ suspect, $4=$ good)". For more details please refer to the User Guide to the Catalogue.
}

also made in prime full window mode with the medium filter. Figure 1 shows the EPIC PN image and the position of GY 141 in the field. GY 141 was not detected in the EPIC MOS2 camera. This source is reported in the XMM-Newton Serendipitous Source Catalogue as 1XMM J162651.3-243242. The catalogue does not report any detection in the EPIC PN camera, although the source appears clearly in it (see Fig. 1). In both EPIC PN and MOS1 the source is very faint, which might explain why the pipeline used to build the catalogue missed it in the EPIC PN image. The corrected detection likelihood value reported in the catalogue for the MOS1 detection is 9.0, with a quality flag of 4. According to the User Guide to the Catalogue, sources with likelihood greater than $\sim 8$ and quality flag of 4 are reliable. GY 141 is just above this limit, which probably explains why it was not detected by the pipeline in the other 
Table 2. X-ray count rates ${ }^{1}$.

\begin{tabular}{lc}
\hline \hline Detector & $\begin{array}{c}\text { Count rates } \\
{\left[10^{-3} \text { counts s }^{-1}\right]}\end{array}$ \\
\hline \multicolumn{2}{c}{ DENIS J155601-233809 } \\
\hline MOS1 & $0.59 \pm 0.20$ \\
MOS2 & Not detected \\
PN & $3.25 \pm 0.49$ \\
MOS1+PN ${ }^{2}$ & $2.48 \pm 0.40$ \\
\hline \multicolumn{2}{c}{ GY 141} \\
\hline MOS1 & $1.66 \pm 0.46$ \\
MOS2 & Not detected \\
PN & $2.73 \pm 0.92$ \\
MOS1+PN ${ }^{2}$ & $2.37 \pm 0.77$ \\
\hline
\end{tabular}

1 Source: XMM-Newton Serendipitous Source Catalogue, except EPIC PN value for GY 141.

${ }^{2}$ Mean total count rate of the detections weighted by the errors.

EPIC detectors. Using an aperture of 320 pixels, 92.6 counts are measured in the EPIC PN image (corrected for background using a standard phot sky algorithm). The corresponding rate is $0.00273 \pm 00092 \mathrm{count} / \mathrm{s}$, which is consistent with the value reported in the catalogue for the EPIC MOS1 camera (see Table 2). The detection in two of the three detectors increases the likelihood that the detection is real, and the chances of coincidence are very low. On the basis of all these considerations, I will consider for the rest of this paper that the detection is real, although the possibility that it is spurious cannot be ruled out.

The X-ray source lies less than 0.'5 away from the DENIS and 2MASS coordinates, and once again no optical/near infrared counterpart can be found in the DENIS and 2MASS catalogues in an area of $\sim 25^{\prime \prime}$ around this position, which makes it very likely that the faint $\mathrm{X}$-ray source is associated with the brown dwarf. As will be discussed in Sect. 4, the associated flux is also consistent with that of a brown dwarf, but higher than the one reported by Imanishi et al. (2001) with Chandra.

\section{Data processing}

Since the source was not centered in the field of view, a correction for vignetting had to be applied using the recommended evigweight task of the XMM-Newton Science Analysis System software (Loiseau 2003). Once this correction was applied, the light curve of each object was extracted in a circular area of 400 pixels around the source, and the light-curve of their respective background in an empty annulus region between 450 and 602 pixels around the source, therefore with the same surface to allow direct comparison. Figure 2 shows the light curves obtained. It is clearly seen that the sources are very faint, i.e. not much brighter than the background. Neither timing nor spectral analysis can therefore reasonably be performed on these data, and I was only able to get flux measurements.

The fluxes reported in the XMM-Newton Serendipitous Source Catalogue were computed for $N_{\mathrm{H}}=3.0 \times 10^{20} \mathrm{~cm}^{2}$, and assuming as a spectral model a power law with a slope of 1.7. For more details about the construction of the catalogue, please refer to the corresponding User Guide ${ }^{2}$. As this spectral model is not the best adapted to this kind of source, the fluxes were recomputed using the count rates reported in the catalogue (see Table 2, except for the PN values of GY 141, measured as explained in Sect. 3.2) and the recommended PIMMS and $n H$ softwares ${ }^{3}$.

Table 3 gives the results obtained assuming a thin thermal plasma with a temperature $k T$ varying from 0.5 to 2.5 , a distance of $145 \mathrm{pc}$ for both objects as measured by de Zeeuw et al. (1999), and bolometric luminosities estimated as explained in Sect. 2.

\section{Data analysis}

Since no spectral analysis could be performed to determine which spectral model best describes the sources, the analysis was done using the results obtained for a thermal plasma with $k T=2.0 \mathrm{keV}$ as shown in Table 3 .

\subsection{DENIS J155601-233809}

As shown in Fig. 3, DENIS J155601-233809 has an X-ray luminosity very similar to that of other late type M-dwarfs in star forming regions and the field. It belongs to the strongest X-ray emitters among that class of objects. It is important to note that no detailed timing analysis can be done because of the very low flux (see Fig. 2), and that more sensitive observations are required to know if the observed emission is quiescent or related to some flare events.

\subsection{GY 141}

The X-ray activity of GY 141 shows that this object has a strong coronal activity. In order to compare the different measurements available, I used the PIMMS software to convert the Chandra luminosity (measured in the energy range $0.5-9.0 \mathrm{keV}$ ) and the ROSAT upper limit (measured in the energy range $0.1-2.4 \mathrm{keV}$ ) to the energy range of the $X M M$-Newton observations $(0.2-12.0 \mathrm{keV})$. The results are summarized in Table 4, and show that the flux of GY 141 increased by a factor of $\sim 14$ between the Chandra and the $X M M$-Newton observations. The X-ray emission is therefore highly variable, and GY 141 would not have been detected in the $33.9 \mathrm{ks} X M M$-Newton frame if its emission had been at the same level as in the $100.6 \mathrm{ks}$ Chandra observation. The present $X M M$-Newton measurement is close to the limit of detection of the ROSAT observation.

As shown in Fig. 3, GY 141 has a very high X-ray luminosity for a late-type object, and displays strong variability between two observations separated by a period of $\sim 10$ months. Neuhäuser et al. (1999) did not detect it with ROSAT in 1994, but their limit of detection was higher than the two values

\footnotetext{
2 XMM-Newton Survey Science Centre Consortium (http://xmmssc-www.star.le.ac.uk/).

3 PIMMS and $n H$ are distributed by the NASA's HEARSAC (http://heasarc.gsfc.nasa.gov/).
} 

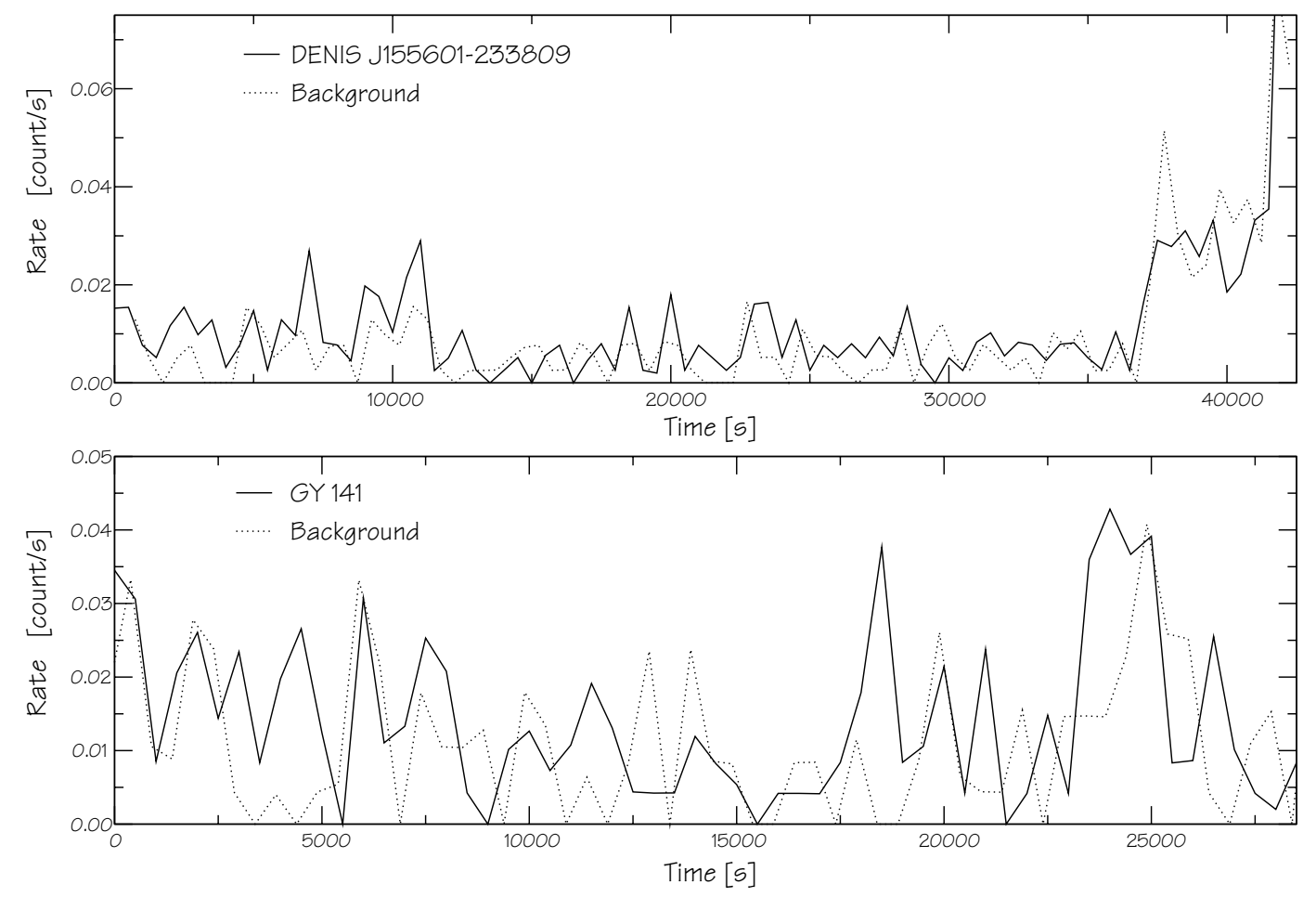

Fig. 2. Light curves of DENIS J155601-233809, GY 141, and the background around their positions. The size of the time bins is $500 \mathrm{~s}$. In both cases the source and the background have very similar amplitudes and variations, indicating that the sources are at the limit of detection. Figure 1 also shows that the two sources are very faint.

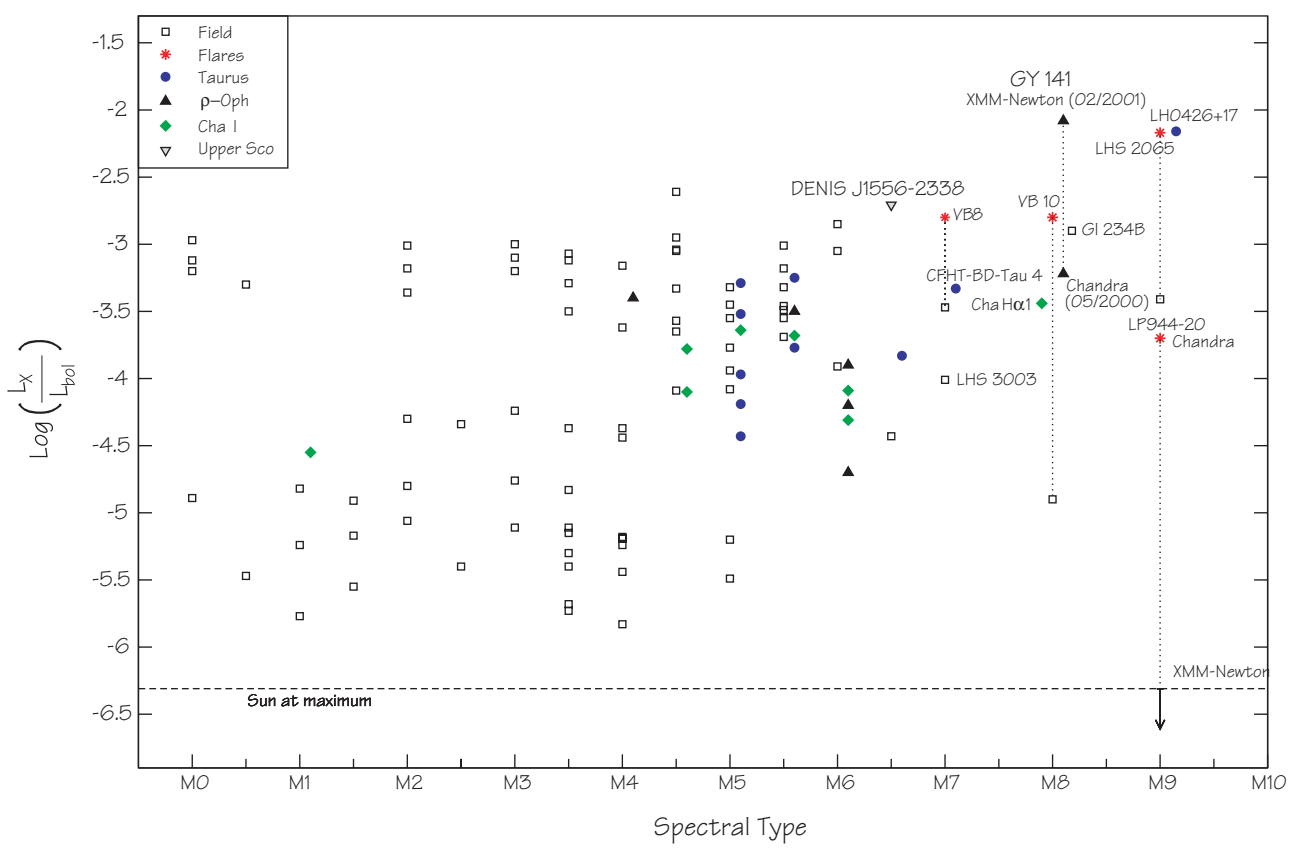

Fig. 3. Distribution of X-ray luminosity of M dwarfs as a function of spectral type. The open squares represent the values obtained for field M-dwarfs. The circles, diamonds, triangles up and down denote young objects in star-forming regions. The stars represent the flaring objects at their maximum with the corresponding value or upper limit on the quiescent emission. While DENIS J155601-233809 seems to have a normal X-ray luminosity in comparison with other M $6 \sim$ M 7 dwarfs, GY 141 shows a strong variability between the Chandra and XMM-Newton observations. To avoid confusion, the spectral types of some objects have been shifted by 0.1 subclass. Values are from Martín \& Bouy (2002) and references therein, except the quiescent value for VB 10, which is from Fleming et al. (2003).

obtained with XMM-Newton and Chandra. Its X-ray luminosity increased by a factor of $\sim 14$ between the Chandra and the XMM-Newton observations. Although the light curve of the emission detected with XMM-Newton suggests that there was no significant variation during the $34 \mathrm{ks} X M M$-Newton observation (see Fig. 2), the shortness of the observation (34 ks) 
Table 3. X-ray flux and luminosities (in the energy range $0.2-12 \mathrm{keV}$ ).

\begin{tabular}{|c|c|c|c|c|}
\hline Detector & $\begin{array}{c}k T^{1} \\
{[\mathrm{keV}]}\end{array}$ & $\begin{array}{c}\text { Flux } \\
{\left[10^{-15} \mathrm{erg} \mathrm{cm}^{-2} \mathrm{~s}^{-1}\right]}\end{array}$ & $\begin{array}{c}L_{\mathrm{X}} \\
{\left[10^{28} \mathrm{erg} \mathrm{s}^{-1}\right]}\end{array}$ & $\log L_{\mathrm{X}} / L_{\mathrm{bol}}$ \\
\hline \multicolumn{5}{|c|}{ DENIS J155601-233809 } \\
\hline \multirow[t]{4}{*}{ MOS1 } & 1.0 & $7.87 \pm 2.67$ & $1.98 \pm 0.67$ & -3.21 \\
\hline & 1.5 & $12.48 \pm 4.24$ & $3.14 \pm 1.06$ & -3.00 \\
\hline & 2.0 & $17.57 \pm 5.96$ & $4.42 \pm 1.50$ & -2.86 \\
\hline & 2.5 & $22.32 \pm 7.58$ & $5.61 \pm 1.91$ & -2.75 \\
\hline \multirow[t]{4}{*}{ PN } & 1.0 & $15.26 \pm 2.32$ & $3.84 \pm 0.58$ & -2.92 \\
\hline & 1.5 & $23.37 \pm 3.55$ & $5.88 \pm 0.89$ & -2.73 \\
\hline & 2.0 & $31.28 \pm 4.75$ & $7.87 \pm 1.19$ & -2.61 \\
\hline & 2.5 & $38.06 \pm 5.77$ & $9.57 \pm 1.45$ & -2.52 \\
\hline \multirow[t]{4}{*}{$\mathrm{MOS} 1+\mathrm{PN}^{2}$} & 1.0 & $12.08 \pm 2.49$ & $3.04 \pm 0.62$ & -3.02 \\
\hline & 1.5 & $18.88 \pm 3.89$ & $4.75 \pm 0.97$ & -2.83 \\
\hline & 2.0 & $25.95 \pm 5.36$ & $6.54 \pm 1.35$ & -2.69 \\
\hline & 2.5 & $32.28 \pm 6.67$ & $8.12 \pm 1.68$ & -2.59 \\
\hline \multicolumn{5}{|c|}{ GY 141} \\
\hline \multirow[t]{4}{*}{ MOS1 } & 1.0 & $22.30 \pm 6.22$ & $5.61 \pm 1.56$ & -2.25 \\
\hline & 1.5 & $35.23 \pm 9.82$ & $8.86 \pm 2.47$ & -2.05 \\
\hline & 2.0 & $49.49 \pm 13.80$ & $12.44 \pm 3.47$ & -1.90 \\
\hline & 2.5 & $62.82 \pm 17.52$ & $15.80 \pm 4.41$ & -1.80 \\
\hline \multirow[t]{4}{*}{ PN } & 1.0 & $13.01 \pm 4.38$ & $3.27 \pm 1.10$ & -2.49 \\
\hline & 1.5 & $19.81 \pm 6.67$ & $4.98 \pm 1.68$ & -2.30 \\
\hline & 2.0 & $26.44 \pm 8.91$ & $6.65 \pm 2.24$ & -2.18 \\
\hline & 2.5 & $32.12 \pm 10.83$ & $8.08 \pm 2.72$ & -2.09 \\
\hline \multirow[t]{4}{*}{$\mathrm{MOS} 1+\mathrm{PN}^{2}$} & 1.0 & $16.09 \pm 5.30$ & $4.05 \pm 1.33$ & -2.39 \\
\hline & 1.5 & $24.67 \pm 8.24$ & $6.21 \pm 2.08$ & -2.20 \\
\hline & 2.0 & $33.22 \pm 11.35$ & $8.35 \pm 2.86$ & -2.07 \\
\hline & 2.5 & $40.61 \pm 14.17$ & $10.21 \pm 3.57$ & -1.99 \\
\hline
\end{tabular}

${ }^{1}$ Temperature of the thermal plasma spectral model used to convert from count rate to flux.

${ }^{2}$ Mean total flux/luminosity of the detections weighted by the errors.

and the faintness of the detection make it possible to conclude that it was not a flare-like event. Further observations should be made to verify if the observed variability occurs on long ( $\sim$ months) or short ( $\sim 10 \mathrm{ks})$ time-scales. This issue is very important since the only few sources known to show variability are flaring objects with periods of $10 \sim 50 \mathrm{ks}$ and, except for the old field brown dwarfs LP944-20 and VB 10, with amplitudes less than a factor of 10 (Imanishi et al. 2003). If confirmed by further observations, a strong variability over a long time-scale would probably mean that two types of activity, governed by different mechanisms, can occur in the corona of late type objects. Recently, Schmitt \& Liefke (2002) reported a significant variation of the quiescent X-ray emission of the M 9 dwarf LHS 2065 after a flare. Such a phenomenon might also be at work and explain the variation observed for GY 141 between the Chandra and XMM-Newton observations. Finally, since it has been shown that the object is surrounded by a disk, part of its emission could also come from the circumstellar material interacting with the magnetic field, following an emission scenario as suggested by Montmerle et al. (2000).

\section{Conclusions}

I report here the detection with XMM-Newton of two young bona-fide brown dwarfs. DENIS J155601-233809 displays an $\mathrm{X}$-ray emission similar to other young objects of the same age and spectral class, with $\log \left(L_{\mathrm{X}} / L_{\mathrm{bol}}\right)=-2.69$. No significant variability was observed during the $48.3 \mathrm{ks}$ observation, but the faintness of the source did not allow me to perform precise timing analysis. More sensitive observations are required to know if this emission is quiescent or associated to a flare-like event. GY 141 has the highest X-ray luminosity reported up to date for that class of object, with $\log \left(L_{\mathrm{X}} / L_{\mathrm{bol}}\right)=-2.07$. Its luminosity has increased by a factor of 14 since the previous Chandra observation. No significant flare-like event could be detected in the $33.9 \mathrm{ks} X M M$-Newton observation. In this case again the 
Table 4. X-ray luminosities $(0.2-12 \mathrm{keV})$ of GY 141 at different epochs.

\begin{tabular}{lccc}
\hline \hline Instrument & Date obs. & $\begin{array}{c}L_{\mathrm{X}} \\
{\left[10^{28} \mathrm{erg} \mathrm{s}^{-1}\right]}\end{array}$ & $\log L_{\mathrm{X}} / L_{\text {bol }}$ \\
\hline ROSAT $^{1}$ & $09 / 1994$ & $\leq 13.00$ & $\leq-1.9$ \\
Chandra $_{\text {XMM-Newton }}$ & $05 / 2000$ & 0.60 & -3.22 \\
XM-N001 & 8.35 & -2.07 \\
\hline
\end{tabular}

${ }^{1} \mathrm{GY} 141$ was not detected. The corresponding value is an upper limit. Remarks: values computed assuming a thermal plasma spectral model with $k T=2 \mathrm{keV}$.

faintness of the source did not allow me to perform precise timing analysis, and more sensitive observations are also required to know if this emission is due to a flare or if it is quiescent. Follow-up observations of this object should allow us to know if this variability occurs on long ( months) or short $(\sim 10 \mathrm{ks})$ time-scales.

Acknowledgements. I would like to thank Elisa Constantini for her precious and generous help regarding the processing of XMM-Newton data. I would like to thank Eduardo Martín and Xavier Delfosse for providing me with the list of Upper Scorpius brown dwarfs prior to publication, and Jérôme Bouvier and Wolfgang Brandner for their kind support.

This research has made use of the XMM-Newton Public Archive and of the XMM-Newton Serendipitous Source Catalogue (XMM-Newton Survey Science Centre 2003). This research has also made use of the VizieR on-line Service at Centre de Donnés astronomiques de Strasbourg, France (Ochsenbein et al. 2000). This publication makes use of data products from the Two Micron All Sky Survey (2MASS) and DEep Near Infrared Survey (DENIS). The Two Mass Survey is a joint project of the University of Massachusetts and the Infrared Processing and Analysis Center/California Institute of Technology, funded by the National Aeronautics and Space Administration and the National Science Foundation. The DENIS project has been partly funded by the SCIENCE and the HCM plans of the European Commission under grants CT920791 and CT940627. It is supported by INSU, MEN and CNRS in France, by the State of BadenWürttemberg in Germany, by DGICYT in Spain, by CNR in Italy, by FFwFBWF in Austria, by FAPESP in Brazil, by OTKA grants F-4239 and F-013990 in Hungary, and by the ESO C\&EE grant A-04-046.

\section{References}

Adams, N., Wolk, S., Walter, F. M., Jeffries, R., \& Naylor, T. 2002, BAAS, 34, 1176
Baraffe, I., Chabrier, G., Allard, F., \& Hauschildt, P. H. 1997, A\&A, 327,1054

Barrado y Navascues, D., \& Martín, E. L. 2003, AJ, in press

Burrows, A., Marley, M., Hubbard, W. B., et al. 1997, ApJ, 491, 856

Comeron, F., Rieke, G. H., Claes, P., Torra, J., \& Laureijs, R. J. 1998, A\&A, 335, 522

Dahn, C. C., Harris, H. C., Vrba, F. J., et al. 2002, AJ, 124, 1170

de Zeeuw, P. T., Hoogerwerf, R., de Bruijne, J. H. J., Brown, A. G. A., \& Blaauw, A. 1999, AJ, 117, 354

Ehle, M., Pollock, A. M. T., Talavera, A., et al. 2003, XMM-Newton Users' Handbook, V2.1

Fleming, T. A. 1998, ApJ, 504, 461

Fleming, T. A., Giampapa, M. S., \& Garza, D. 2003, ApJ, 594, 982

Fleming, T. A., Giampapa, M. S., \& Schmitt, J. H. M. M. 2000, ApJ, 533,372

Gorlova, N. I., Meyer, M. R., Rieke, G. H., \& Liebert, J. 2003, ApJ, 593,1074

Imanishi, K., Tsujimoto, M., \& Koyama, K. 2001, ApJ, 563, 361

Imanishi, K., Nakajima, H., Tsujimoto, M., Koyama, K., \& Tsuboi, Y. 2003, PASJ, 55, 653

Jayawardhana, R., Mohanty, S., \& Basri, G. 2002, ApJ, 578, L141

Jayawardhana, R., Mohanty, S., \& Basri, G. 2003, ApJ, 592, 282

Küker, M., \& Rüdiger, G. 1999, A\&A, 346, 922

Leggett, S. K., Golimowski, D. A., Fan, X., et al. 2002, ApJ, 564, 452

Loiseau, N. 2003, XMM-Newton Science Analysis System Users' Guide, V2.1

Luhman, K. L., Liebert, J., \& Rieke, G. H. 1997, ApJ, 489, L165+

Martín, E. L., \& Bouy, H. 2002, New Astron., 7, 595

Martín, E. L., Delfosse, X., \& Guieu, S. 2004, AJ, accepted

Mokler, F., \& Stelzer, B. 2002, A\&A, 391, 1025

Montmerle, T., Grosso, N., Tsuboi, Y., \& Koyama, K. 2000, ApJ, 532, 1097

Neuhauser, R., \& Comeron, F. 1998, Science, 282, 83

Neuhäuser, R., Briceño, C., Comerón, F., et al. 1999, A\&A, 343, 883

Ochsenbein, F., Bauer, P., \& Marcout, J. 2000, A\&AS, 143, 23

Preibisch, T., \& Zinnecker, H. 2002, AJ, 123, 1613

Rutledge, R. E., Basri, G., Martín, E. L., \& Bildsten, L. 2000, ApJ, 538, L141

Schmitt, J. H. M. M., \& Liefke, C. 2002, A\&A, 382, L9

Stelzer, B. \& Neuhäuser, R. 2003, in Brown Dwarfs, Proc. IAU Symp., 211, held 20-24 May 2002 at University of Hawai'i, Honolulu, Hawai'i, ed. E. Martín, 443

Tsuboi, Y., Maeda, Y., Feigelson, E. D., et al. 2003, ApJ, 587, L51

Wolk, S. J. 2003, in Brown Dwarfs, Proc. IAU Symp., 211, held 20-24 May 2002 at University of Hawai'i, Honolulu, Hawai'i, ed. E. Martín, 447

XMM-Newton Survey Science Centre 2003, The First XMM-Newton Serendipitous Source Catalogue 